\title{
Belphégor
}

Lorsque peinture, littérature et musique suivent la piste des sirènes.

L'imaginaire océanique dans De Profundis, album interactif de Miguelanxo Prado

\section{Catherine d'Humières}

\section{CpenEdition}

Journals

Édition électronique

URL : http://journals.openedition.org/belphegor/447

DOI : $10.4000 /$ belphegor.447

ISSN : 1499-7185

Éditeur

LPCM

Référence électronique

Catherine d'Humières, « Lorsque peinture, littérature et musique suivent la piste des sirènes.

L'imaginaire océanique dans De Profundis, album interactif de Miguelanxo Prado », Belphégor [En ligne], 12-1 | 2014, mis en ligne le 15 juin 2014, consulté le 19 avril 2019. URL : http:// journals.openedition.org/belphegor/447 ; DOI : 10.4000/belphegor.447

Ce document a été généré automatiquement le 19 avril 2019.

\section{(†) $\odot$

Belphégor est mis à disposition selon les termes de la Licence Creative Commons Attribution - Pas d'Utilisation Commerciale - Pas de Modification 4.0 International. 


\title{
Lorsque peinture, littérature et musique
} suivent la piste des sirènes. L'imaginaire océanique dans De Profundis, album interactif de Miguelanxo Prado

\author{
Catherine d'Humières
}

1 Un îlot perdu au milieu de l'océan avec juste une maison et un arbre pour marquer le passage des saisons et, dans cette maison, amoureux l'un de l'autre, une violoncelliste qui fait danser les baleines et un peintre qui rêve d'être marin et pêcheur au risque d'en mourir.

2 Dans l'album De Profundis, de l'Espagnol Miguelanxo Prado, la peinture, la musique et la littérature s'unissent pour évoquer le mystérieux monde des océans, à la fois sujet d'observation artistique, tellement les grands fonds recèlent de trésors insoupçonnés, et source d'inspiration fantastique et onirique, royaume des sirènes éternelles et demeure précaire des âmes des marins naufragés avant leur ultime transfiguration ${ }^{1}$.

3 De Profundis (Norma, 2008) est un produit complet et complexe : à la fois album et film d'animation, il est destiné à tous publics et fait appel à trois de nos sens : la vue, bien entendu, en ce qui concerne les deux supports, et également l'ouie pour la très belle musique du film, et le toucher dans le cas de l'ouvrage que l'on feuillette avec délice. À ces trois sens, et seulement si l'on accepte d'entrer dans le monde que nous propose l'auteur, on peut ajouter l'odorat et le goût tellement l'océan est partie prenante de toute l'histoire. Dans L'Eau et les rêves, Gaston Bachelard souligne combien l'eau est la maitresse $\mathrm{du}$ langage fluide, du langage sans heurt, du langage continu, continué, du langage qui assouplit le rythme, qui donne une matière uniforme à des rythmes différents " (Bachelard : 209). L'étude de l'ensemble artistique composé par Miguelanxo Prado, et dont la fluidité est certainement l'une des caractéristiques les plus importantes, nous 
permettra d'envisager la place tenue par le mélange des formes d'expressions que sont la peinture, l'écriture, la musique et le cinéma, non seulement dans l'évocation d'un monde naturel rendu fantastique par l'imagination de l'artiste, mais également dans le déroulement d'une histoire dont l'écho résonne longtemps dans l'esprit du lecteurspectateur.

Dans leur ouvrage sur Les Frontières du fantastique, Roger Bozzetto et Arnaud Huftier soulignent combien ces dernières "apparaissent friables, poreuses et mouvantes " (Bozzetto, Huftier : 7). Il n'est pas question, pour nous, de revenir ici sur la différence tant étudiée entre le fantastique et le merveilleux, présents dans l'album dont nous allons parler, puisqu'il se situe justement sur cette incertaine frontière, mais nous désirons faire nôtre l'affirmation selon laquelle les effets de fantastique " traduisent le rapport de l'être au monde » (Ibid. : 45), et sont «l'ombre portée de la littérature [...et] aussi l'ombre de l'homme » (Ibid. : 47). En effet, comme on le verra, ce sont bien les éléments naturels, rendus fantastiques par l'imagination artistique de l'auteur, qui donnent une ampleur inattendue à l'aventure humaine.

C'est pourquoi, après avoir analysé l'importance du rôle de la nature dans la création artistique et montré comment les éléments fantastiques issus de cette dernière restent tributaires de l'offre multiforme de la première, nous envisagerons l'influence de légendes atlantiques issues de l'esprit des populations qui vivent au bord du grand océan - populations fascinées et terrorisées par des forces naturelles indomptables et redoutables - et qui expriment sous forme de récits fabuleux leurs inquiétudes et leurs interrogations transcendentales.

\section{La nature, source de création artistique}

Selon Valérie Tritter, «le fantastique se présente comme une forme mouvante capable d'investir tous les genres littéraires existants, mais certains de manière plus efficace quant à l'effet produit, que d'autres " (Tritter: 37). Cette affirmation, qui aboutit à énumérer la poésie, le théâtre et le genre romanesque incluant le roman et la nouvelle comme uniques genres littéraires existants, laisse étrangement de côté les formes nouvelles que sont les productions illustrées comme l'album. Et pourtant, vu l'abondance et la variété de la production actuelle, il s'agit bien d'un nouveau genre littéraire ${ }^{2}$, capable de transcender les frontières du merveilleux, du fantastique et du réel pour proposer des créations originales, souvent d'une extrême beauté artistique.

7 Miguelanxo Prado est un peintre galicien et c'est un élément d'importance pour comprendre ce qui fait l'essence de De Profundis. En effet, la Galice forme l'extrémité nordouest de l'Espagne dont les côtes sont découpées de très profondes rías, estuaires qui s'avancent loin dans les terres et sont soumis aux fortes marées océaniques. C'est une région à fort particularisme, ancienne terre celte, entièrement tournée vers l'océan Atlantique, et tributaire de ses fureurs et de ses richesses. La pêche a toujours été une activité importante en Galice et l'extrême variété de la faune marine a fait la réputation de sa gastronomie. Il n'est donc guère étonnant que les œuvres d'un peintre galicien cherchent à refléter la fascination éprouvée pour l'univers maritime. À la fin de l'album, dans le DVD des « extras », il y a un très long documentaire de Miguelanxo Prado, intitulé « Rêves du fond de l'abîme » - «Sueños desde el abismo »-, référence directe aux premiers mots du psaume 129: «De Profundis clamavi ad te, Dómine... ", d'où procède le titre de l'album. Dans ce documentaire, l'auteur évoque à la fois les sources de son inspiration et 
les étapes de l'élaboration de l'album et du film. Habitant La Corogne, ville entourée d'eau de tous côtés ${ }^{3}$, sa peinture est basée sur l'observation de ce contexte océanique : une luminosité particulière, des oiseaux volant dans le vent, des cétacés jouant dans les vagues, des îles, des phares, et le mouvement inlassable des eaux couleur «d'émeraude sombre »- «esmeralda oscuro »- (Prado: 21). De même, la pêche étant l'une des principales activités de la ville, le monde des pêcheurs, leurs bateaux, leur travail, lui sont familiers, et c'est au marché aux poissons qu'il va observer et photographier les poissons et fruits de mer encore vivants, fraichement pêchés, afin de compléter l'observation de la faune marine et de pouvoir s'appuyer sur le monde naturel pour nourrir son inspiration artistique.

8 Mais l'observation de l'océan demeurerait source de frustration sans le pouvoir de l'imagination car les fonds marins restent encore en grande partie inexplorés. Pour Miguelanxo Prado, ce qu'on voit de la mer, c'est son aspect extérieur, sa peau en quelque sorte. Et l'on sait finalement peu de choses sur ce qui se passe en dessous, ce qui laisse la voie libre à l'inspiration artistique. À la fin de l'album, se trouvent quelques pages appelées «Carnet de bord »- «Cuaderno de bitácora »- et qui sont en réalité des pages d'esquisses fort intéressantes en ce qu'elles mélangent l'observation du réel et sa transfiguration fantastique. C'est également bien visible dans l'une des séquences du film où un pêcheur regarde d'un air dubitatif le peintre en train de dessiner l'un des poissons qu'ils viennent de pêcher, tellement la création artistique lui paraît éloignée du produit de la nature. Dans le DVD des "extras ", le chapitre «Incognita natura " se présente comme un album de dessins de la faune océanique de De Profundis, inventée à partir d'animaux connus, comme si l'acte créateur de l'artiste voulait s'inscrire dans la normalité en proposant une nouvelle anthologie de créatures aquatiques nées de son imagination. L'existence des méduses, par exemple, lui permet d'imaginer la merveilleuse Acquamaris regia, celle des poissons volants a servi d'inspiration pour les fragiles poissonslibellules, et celle des conques pour le coquillage-fleur ou le coquillage-escargot. Les raies deviennent de merveilleux papillons sous-marins et les étoiles de mer recouvrent le fond de l'océan en un parterre multicolore. On notera d'ailleurs que ce principe créatif d'exaltation fantastique d'éléments naturels choisis dans ce but sera également mis en œuvre dans le très bel album de Benjamin Lacombe et Sébastien Pérez, L'Herbier des fées, destiné aux enfants à partir de huit ans et publié en 2011 chez Albin Michel.

Le même processus de transfiguration de la nature pour créer un monde imaginaire, ou plutôt pour accompagner le récit fantastique créé par Miguelanxo Prado, se retrouve dans la musique symphonique du compositeur Nani García, écrite de façon à entremêler le son mélodique des instruments, le bruit des vagues et du ressac, le cri des mouettes ou le chant mystérieux des baleines, avec même parfois de précieux moments de silence. En effet, comme l'affirme Bachelard, il n'y a "pas de grande poésie [...] sans de larges intervalles de détente et de lenteur, pas de grands poèmes sans silence » (Bachelard : 215). Certes, l'histoire ne pourrait pas se dérouler sans la fureur de l'océan mais, la plupart du temps, c'est la poésie du silence et la douceur du monde aquatique qui s'imposent : « l'eau est un modèle de calme et de silence " (Ibid.) et, pour Miguelanxo Prado, image et musique sont étroitement liées. Nous ne prendrons comme exemple que les scènes du début qui semblent renvoyer l'écho d'un bonheur quotidien mais sont, en même temps, des images fantastiques tellement cette maison et cet arbre plantés tout seuls sur leur îlot se présentent comme une porte ouverte sur l'imaginaire. La musique de ces premières scènes accompagne cette douceur, la présence de la violoncelliste se fait enchantement et 
on ne s'étonne plus alors de voir danser des baleines mélomanes. Ici, le monde naturel est omniprésent dans l'expression artistique, mais il est sublimé de façon à entrouvrir les portes $\mathrm{du}$ fantastique pour un lecteur-spectateur prisonnier consentant de cette fantasmagorie. Le meilleur exemple est peut-être celui des cétacés, très présents dans l'œuvre de Miguelanxo Prado, car on les retrouve aux trois niveaux de l'expression artistique : dans le texte, dans l'image et dans la musique puisque leur chant possède justement une résonance étrange, presque magique, qui rend leur présence essentielle dans le déroulement de l'histoire.

\section{La nature, source d'inspiration poétique}

10 La Galice étant entourée au nord et à l'ouest par l'Océan Atlantique, c'est l'imaginaire nordique qui prévaut sur l'imaginaire méditerranéen auquel on a tendance à rattacher l'Espagne de façon exclusive. C'est pourquoi nous avons choisi de faire appel à l'évocation que fait Andersen des fonds sous-marins dans «La petite Sirène ». On peut, en effet, trouver de nombreux points communs entre son inspiration poétique et celle de Prado :

Mais n'allez pas croire que ce fond se compose seulement de sable blanc; non, il y croît des plantes et des arbres bizarres, et si souples que le moindre mouvement de l'eau les fait s'agiter comme s'ils étaient vivants. Tous les poissons, grands et petits, vont et viennent entre les branches comme les oiseaux dans l'air. À l'endroit le plus profond se trouve le château du roi de la mer, dont les murs sont de corail, les fenêtres de bel ambre jaune, et le toit de coquillages qui s'ouvrent et se ferment pour recevoir l'eau et la rejeter. Chacun de ces coquillages renferme des perles brillantes dont la moindre ferait honneur à la couronne d'une reine.

(Andersen : 230)

11 On observera que le merveilleux de ce conte si connu s'appuie intensément - et poétiquement - sur la description de la nature du fond des mers et des paysages côtiers. La transfiguration fantastique à l'œuvre dans De Profundis, procède de la même vision poétique, et la métamorphose du protagoniste est également au cœur du conte même si elle prend une tout autre forme: le peintre, noyé au cours d'une tempête, renaît mystérieusement, retrouve l'épave du bateau-pêcheur dans lequel il avait pris place, lui redonne vie, se fond en lui et se transforme progressivement en « un cétacé splendide, aux couleurs invraisemblables »- « un cetáceo espléndido de colores imposibles »- (Prado : 76) qui viendra danser son amour pour la violoncelliste devant la maison qui avait abrité leur bonheur conjugal.

Ce schéma narratif correspond bien à celui que décrit Christophe Carlier :

Deux mondes ici se font face. A la durée appartiennent les premières lignes du conte. [...] L'action elle-même naît avec le bouleversement de cet état de fait. Elle introduit rupture, déséquilibre et péril. Le dénouement vient proposer un nouvel équilibre dont on comprend bien qu'il sera durable. Le lecteur, initialement installé dans la durée, quitte un instant ce monde immobile. Il se livre aux imprévisibles soubresauts de la succession, et renoue pour finir avec la durée.

(Carlier : 43)

12 Les premières pages de l'album inscrivent effectivement dans la durée la maison, l'art et l'amour, qui reviennent à la fin de l'histoire, avec plus de force encore, exaltés au maximum pour s'inscrire dans un désir d'éternité joyeuse. Entretemps, ce bonheur tranquille, cette harmonie paisible auront été rompus par la tempête, le naufrage, la noyade, la renaissance et le retour, tout ce qui aura composé le récit lui-même. Il n'en est pas moins vrai que la maison dans l'île, hybride d'eau et de terre finalement, est elle- 
même habitée par le fantastique, dans la mesure où, pour l'auteur, les cadres du peintre, protagoniste de l'histoire,

ouvraient dans les murs des fenêtres secrètes vers les eaux silencieuses de ces profondeurs imaginées, transformant la maison en métaphore de cette ligne très fine et imprécise qui marque parfois la frontière entre la réalité - la mer véritable qui l'entoure - et le rêve - la Mer imaginée qui emplit son intérieur-.

abrían en las paredes ventanas secretas a las aguas silentes de aquellas profundidades imaginadadas, convirtiendo la casa en metáfora de esa finísima e imprecisa frontera entre la realdidad - el mar verdadero que la rodeaba- y el ensueño-el Mar imaginado que llenaba su interior -.

(Prado: 5)

13 Nous noterons la majuscule de la Mer dans le second cas, celui du rêve: l'écriture poétique la magnifie, la personnifie, et contribue à la transfigurer par la magie de l'écriture en un être fécond et mystérieux à qui l'on peut attribuer tout un monde grouillant de créatures fantastiques, largement imaginaires. Pour Miguelanxo Prado - et il l'explique clairement dans "Sueños desde el abismo »- le contact avec la nature est essentiel car elle est partie prenante de sa façon de sentir, de voir et de penser le monde, et sa présence est constante dans ses œuvres. Néanmoins, chez lui, la certitude que tout est dans la nature s'accompagne de celle que rien n'est donné gratuitement et qu'il faut une vraie recherche pour faire éclore les germes de l'imagination qui produira l'œuvre littéraire. Il épouse ainsi l'idée de Michel-Ange qui expliquait que sculpter consistait à « enlever le superflu » - « togliere il superfluo » - comme si la sculpture était déjà en germe dans le bloc de marbre brut et que seul le génie de l'artiste pouvait la faire émerger. Il est d'ailleurs significatif que l'auteur ait attribué un nom savant aux créatures fantastiques qu'il a imaginées à partir de son observation du monde animal alors que ses deux protagonistes humains n'ont pas été dotés de noms propres. D'après C. Carlier,

Ce balancement entre le monde connu et la Terra incognita définit une géographie imaginaire dont les composantes sont tout à la fois déconcertantes et rituelles. [...] Ces lieux sont-ils irréels? Il est permis d'en douter. La description de leur découverte marque fortement le lien entre notre monde et ces terres chimériques et dit assez ce qui est en jeu. Parfois, le symbolisme identifie à la mort le passage d'un monde à l'autre. À l'occasion [...] Edgar Poe [peut en tirer] une variation onirique sur l'au-delà. Dans les Aventures d'Arthur Gordon Pym, il égare son héros dans une géographie abyssale. Le conte fantastique use pleinement de ce procédé. (Carlier :36-37)

C'est bien ce qui est en jeu dans De Profundis, où l'auteur propose une espèce de réflexion sur une possible métempsycose en imaginant l'étrange transmigration du personnage principal en cétacé à partir du moment où il s'empare de l'épave du bateau de pêche, emblématique d'un désir caché et imparfaitement réalisé. Il faut, pour entrer vraiment dans l'histoire, que le lecteur accepte le pacte de lecture proposé par l'auteur: l'idée, récurrente dans bien des récits merveilleux ou fantastiques, que l'Homme est susceptible de se transformer en une autre créature, comme par exemple dans La Métamorphose de Kafka, lorsque Grégoire Samsa se réveille scarabée. L'imagination littéraire abolit de la sorte les frontières naturelles entre les espèces et, tout en s'appuyant sur le monde naturel connu, n'en finit pas de faire montre de son extrême créativité. C'est pour cela qu'il n'est guère étonnant que le protagoniste de De Profundis rencontre des êtres hybrides d'hommes et de bêtes dans les fonds sous-marins et qu'il puisse lui-même renaître sous la forme d'un cétacé, ramenant ainsi le lecteur à une hypothétique condition aquatique originelle. 


\section{La nature, mère des mythes et des légendes} grand'père, le monde de la mer est un monde magique et dangereux, et celui des profondeurs océaniques à la fois plein d'attraits et d'angoisse. On peut rapprocher ce point de vue de celui exprimé par Jules Michelet dans La Mer, où il « montre [...] la place et la signification de la mer dans la vie humaine en soulignant tout d'abord que la mer est l'espace de la vie, le berceau de la vie terrestre » (Malgorzata Wierbowka : 69). En effet, l'univers artistique de Miguelanxo Prado est empli de rêves et de cauchemars liés à cet environnement océanique - « le Père Océan » / «Padre Océano » - et son œuvre se nourrit de symboles et de représentations en accord avec des traditions très anciennes qui confondent toujours le réel et le fantastique dans une tension constante vers un impossible paradis perdu.

C'est de là que sont issus le légendaire poisson maléfique "qui s'alimente des âmes des marins morts en mer sans que personne ne se souvienne d'eux » - «que se alimenta de las almas de los marineros que mueren en el mar sin que nadie los recuerde »- (Prado: 17), ainsi que l'ondin et la sirène présents dans De Profundis. Cette dernière est fort éloignée de la tradition mythique gréco-latine où les sirènes dévoratrices du golfe de Sorrente, sans doute divinités des promontoires à l'origine, étaient l'incarnation des dangers de ceux-ci ${ }^{4}$. Elle aurait davantage à voir avec les sœurs de celle d'Andersen dans la mesure où elle est heureuse dans son univers aquatique et ne rêve pas d'en sortir, même par amour, contrairement à l'héroïne du conte. Elle serait finalement plutôt en phase avec une autre tradition mythique et légendaire, plus nordique et plus océanique, peut-être celtique, où la sirène, pourtant également liée aux mystères de la vie et de la mort comme, finalement, toutes les sirènes, représente surtout la beauté des fonds sous-marins qui constituent son royaume. Cette tradition peut être maléfique dans la mesure où elle a donné naissance à des sirènes désireuses d'entraîner chez elles, et donc dans la mort, un homme incapable de vivre sans air mais, dans cet album, la sirène est plutôt l'hôtesse qui accueille le peintre au terme de sa noyade, et lui fait découvrir l'ampleur des merveilles que recèle le monde sous-marin qui se déploie devant eux. Elle sert de passeur, c'est un être psychopompe qui emporte l'âme du mort vers une autre vie. En effet, après lui avoir fait suivre un parcours jalonné de beautés naturelles, elle se détache de lui lorsqu'il franchit le seuil énigmatique au delà duquel l'attend une autre vie, et elle le laisse assumer lui-même la métamorphose qui le mène vers un autre destin. Cette sirène est issue des peintures à l'huile de Miguelanxo Prado qui ornent la maison sur l'île et dont nous avons déjà vu qu'elles étaient comme autant de fenêtres ouvertes sur un monde sous-marin imaginaire et fantasmé, dessiné à partir de la beauté naturelle, connue et supposée, des créatures sous-marines. Cela conforte l'opinion de Valérie Tritter, selon laquelle :

Il n'est pas rare de trouver l'œuvre d'art ou l'art lui-même à différents degrés présents au cœur du récit fantastique : tantôt comme thème spectaculaire, pierre de touche d'une intrigue, tantôt comme thème spéculaire, matière à réflexion, le texte faisant un retour sur lui-même et interrogeant sa qualité d'œuvre d'art. (Tritter : 82)

Ici, tout le récit fonctionne comme si le naufrage et la noyade avaient transporté le peintre au cœur même de ses productions artistiques, comme si le monde naturel fantastique qu'il découvre au fond des mers était celui qu'il portait déjà au fond de lui. 
Dans les «extras ", l'auteur explique que, à son avis, les produits de l'imagination humaine viendraient de souvenirs enfouis, de sensations altérées par un oubli partiel. Comme si le pouvoir créateur de l'artiste et sa capacité de translation dans un monde fantastique étaient liés à la présence d'une mémoire archaïque qui ramènerait l'homme vers une époque très antérieure où il était créature aquatique, à l'aise dans un monde marin dont il garderait, profondément enfoui en lui, une certaine nostalgie mêlée à une profonde angoisse. Ces deux sentiments se traduiraient par des rêves étranges et récurrents, pas vraiment des cauchemars, mais des rêves qui transmettraient à la fois un profond désir de submersion dans l'élément liquide, les eaux originelles, et une forte sensation d'inquiétude face à l'inconnu que représente le monde des profondeurs. Cela permettrait, selon lui, d'expliquer l'attirance qu'exerce la mer sur beaucoup d'hommes, attirance qu'il met en scène dans son album :

Ce peintre, comme tant d'autres poètes et peintres, comme tant d'autres êtres humains, était convaincu d'avoir une âme de marin, car il ne parvenait pas à expliquer autrement cette fascination pour la Mer et, lors de nombreux crépuscules, accompagné des notes vibrantes du violoncelle entremêlées à la rumeur infinie des vagues, il s'appliqua à rêver un bateau sur lequel un beau jour il naviguerait sur le Père Océan.

Aquel pintor, como tantos otros poetas y pintores, como tantos otros seres humanos, estaba convencido de tener alma de marinero, pues no conseguía de otra forma explicar aquella fascinación por el Mar, y durante muchos anocheceres, con las notas vibrantes del violonchelo entretejidas con el rumor infinito del oleaje, se aplicó a soñar un barco con el que algún día navegaría todo el Padre-Océano. (Prado: 6)

On comprend bien alors comment l'artiste a pu trouver normal de transformer son bateau rêvé, une fois habité par l'âme du peintre-marin, en un navire-cétacé capable d'aller danser sa joie de re-vivre au son de l'inoubliable musique de sa bien-aimée.

Si l'étude de cet ensemble, album et film d'animation, nous a paru intéressante, c'est par l'union étroite du naturel et du fantastique qui fonde la fiction en un va-et-vient subjectif et profondément créateur. Miguelanxo Prado, en s'appuyant sur l'observation d'un univers maritime empreint de quotidien pour élaborer un conte fabuleux en plusieurs dimensions, mêle la profondeur des mystères océaniques à ceux de la mort et de l'amour. Pour lui, « De profundis est un poème visuel et musical. C'est aussi une histoire d'amour étrange et fantastique. Et c'est un voyage créatif de peintre, un jeu de métaphores. C'est un hommage à la Mer, la vraie mer et ses qualités mythiques. ${ }^{5}$ La narration, en quittant l'univers du littéraire pur pour intégrer d'autres formes d'expression artistique, exalte un monde naturel où la réalité et la fiction nourrissent à parts égales les rêves profonds de l'Homme et réalisent son extrême désir de transcendance en lui proposant une renaissance d'amour et de beauté.

\section{BIBLIOGRAPHIE}

\section{Ouvrages de référence}

Prado, Miguelanxo. De Profundis. Barcelona (Espagne). Norma, 2008 
Andersen, Hans Christian. « La Petite Sirène » in Contes d'Andersen, traduits du danois par D. Soldi. Paris : Hachette, 1862

\section{Études et critiques}

Bachelard, Gaston. L’Eau et les rêves. Paris : Librairie José Corti, 2010 (1ère édition : 1942).

Bozzetto, Roger et Huftier, Arnaud. Les Frontières du fantastique. Valenciennes (France) : Presses Universitaires de Valenciennes, 2004.

Carlier, Christophe. La clef des contes. Paris : Ellipses, « Thèmes et études », 1998.

Humières, Catherine (d'). « La sirène dans la littérature européenne : d'un imaginaire mythique à l'autre ", in Figures mythiques. Fabrique et métamorphoses, direction V. Léonard-Roques. ClermontFerrand (France) : Presses Universitaires Blaise-Pascal, 2008.

Khoury, Jorge. Pop - 4/26. Comic House. Comic book Resources : 1995-2012. <http:// www.comicbookresources.com/print.php?type=ar\&id=20963>

Malgorzata Wierzbowka, Eva. « Homo aquaticus. La Mer de Jules Michelet », in In Aqua Scribis. Le thème de l'eau dans la littérature, direction M. Piotr Mrozowicki. Gdansk (Pologne) : Wydawnictwo Uniwersytetu Gdanskiego, 2005.

Serrano, José A. « Miguelanxo Prado » Guía del cómic.es. 2003-2010. <http :/guiadelcomic.es/ miguelanxo-prado/index.htm>

Tritter, Valérie. Le fantastique. Paris : Ellipses, « Thèmes et études », 2001.

\section{NOTES}

1. N'ayant pu obtenir l'autorisation de reproduction des illustrations de l'album, nous conseillons au lecteur de regarder la bande annonce du film sur : http://www.youtube.com/watch? $\mathrm{v}=\mathrm{vyhakiu} 4 \mathrm{ftE}$ ou avec les mots-clefs : De profundis trailer.

2. Comme souvent en ce qui concerne les productions modernes qui mélangent les genres, on trouve peu de véritables études ou de critiques de l'œuvre de Miguelanxo Prado.

3. Le centre de La Corogne occupe une péninsule reliée au continent par un isthme très étroit qui présente deux façades maritimes différentes : le port d'un côté et la mer de l'autre, c'est de ce côté-là que s'étendent les principales plages.

4. Voir à ce propos notre article : « La sirène dans la littérature européenne : d'un imaginaire mythique à l'autre ", in Figures mythiques. Fabrique et métamorphoses. Clermont-Ferrand: PUBP, 2008.

5. «De profundis is a visual and musical poem. It is also a history of love that is strange and fantastic. And it is a creative voyage of a painter, a game of metaphors. It is an hommage to the Sea, the real sea and its mythical qualities. » Miguelanxo Prado lors d'un entretien avec Jorge Khoury pour Pop - 4/26. Comic House. Comic book Resources:1995-2012, pp.10-11. 


\section{AUTEUR}

\section{CATHERINE D'HUMIÈRES}

Maître de conférences à l'Université de Cergy-Pontoise, Espagnol, langue et didactique.

Membre statutaire du CRTF (Centre de recherches Textes et Francophonie) de l'Université de Cergy-Pontoise, membre associé du CELIS (Centre d'Études des Littératures et de la Sociopoétique) de l'Université Blaise-Pascal, de Clermont-Ferrand.

Axes de recherches: Littérature comparée des langues romanes (espagnol, français, italien, portugais). Sociopoétique des Mythes dans les littératures de langue romane et dans les productions pour la jeunesse - $\mathrm{XX}^{\mathrm{e}}-\mathrm{XXI}^{\mathrm{e}}$ siècles.

Parmi ses publications: D'un conte à l'autre. D’une génération à l'autre, ouvrage collectif dirigé par Catherine d'Humières, Clermont-Fd, PUBP, « Littératures », 2008. « Sur le modèle du labyrinthe, lorsque la littérature privilégie le jeu », in Amaltea. Revista de mitocrítica vol. 1 (2009) pp. 133-144. http://www.ucm.es/info/amaltea:revista/fr/num1.html "Monstrueuses ténèbres : Beltenebros d'Antonio Muñoz Molina ", in Le monstre (Espagne \& Amérique Latine), dir. Francis Desvois, Paris, L’Harmattan, 2009, pp. 533-547.

«Une petite place dans la crèche ? Les animaux dans les albums de Noël pour enfants au XXI siècle ", in Présent(s) de Noël en littérature de jeunesse contemporaine, dirigé par Danièle Henky et Robert Hurley, Novalis, Québec (Canada), 2010, pp. 39-60. Version téléchargeable sur :www.novalis.ca/client/images/nouveau_site/fr/documents/

ARTICLE_DHUMIERES_NOVALIS.pdf 